\section{P72 WHAT IS GOOD END OF LIFE CARE AFTER STROKE?}

Clare Jeffries, ${ }^{1}$ Cathy Shipman, ${ }^{2}$ Bee Wee ${ }^{3}{ }^{1}$ Marie Curie Hospice, Liverpool, UK ${ }^{2}$ Department of Palliative Care, King's College London, UK: ${ }^{3}$ Sir Michael Sobell House, Oxford, UK

10.1136/bmjspcare-2011-000105.72

Introduction Stroke is the third commonest cause of death and causes significant symptoms and disability. Acute stroke care in the UK predominantly occurs in hospital. Guidelines recommend that patients should be cared for in stroke units to improve survival and disability.

The End of Life Care (EOLC) Strategy aims to improve care for all irrespective of diagnosis and place of care. Previous research suggests that stroke patients and families have unmet palliative care needs. Researching these needs presents challenges due to the effect of stroke on communication, cognition and consciousness.

\title{
Aims
}

1. To determine what healthcare professionals on a stroke unit believe constitutes good EOLC for stroke patients.

2. To determine any barriers exist to implementing good care.

Methods 15 healthcare workers from an acute stroke unit were purposively sampled. Semi-structured interviews were recorded, transcribed and analysed using framework analysis.

Results Professionals believed the following were important aspects of end of life care:

- Treating patients as individuals

- Providing care in a dignified manner and in a comfortable environment

- Good personal care and symptom control

- Ascertaining the needs and monitoring patients

- Recognising death

- For the team to be aware of the care plan

- Informing families and involving them in decisions

- Facilitating patient communication and patient-family interaction was also valued

- Good team structure, function and training.

Barriers to care included resource and time limitation, competing priorities, difficulties in prognostication and communication and not knowing patients' wishes.

Conclusion Importantaspects of care are identified. Healthcare professionals' views led to recommendations which include:

- Using a care of the dying pathway

- Encouraging patients with capacity to discuss their care needs and wishes 
- Discussion of dying patients care at multidisciplinary meetings

- Facilitating patient communication

- Educating stroke team in palliative care and palliative care teams in stroke care

- Research into prognostication is needed. 